\title{
Aetiopathology of rheumatoid arthritis
}

Peter C Taylor

Nehal Narayan

Peter C Taylor MA PhD FRCP FRCPE is the Norman Collisson Professor of Musculoskeletal Sciences, University of Oxford and a Fellow of St Peter's College Oxford, UK. His clinical expertise is in rheumatoid arthritis, ankylosing spondylitis and primary inflammatory arthritis. He is Head of Clinical Sciences at the Botnar Research Centre, Nuffield Dept of Orthopaedics, Rheumatology and Musculoskeletal Sciences. Competing interests: Professor Taylor has received research grant support from UCB Pharma, Janssen, Celgene and Lilly. He has served as a consultant for UCB Pharma, Merck, Pfizer, Lilly, Biogen, Sandoz, Novartis, Roche, BMS, Janssen and Abbvie.

Nehal Narayan MB BS BSc MRCP (Rheumatology) is an ST5 Rheumatology registrar in the West Midlands UK, currently undertaking out-of-programme research as a DPhil student at the University of Oxford, UK. Competing interests: none declared.

\begin{abstract}
Rheumatoid arthritis (RA) has a multifactorial aetiopathogenesis with both genetic and environmental factors implicated, giving rise to immune dysregulation with resultant joint inflammation and tissue damage. The emergence of biologic and small molecular targeted therapeutics has validated the role of many key molecules and cells in the pathogenesis of RA. The major cellular components, cytokines and key signalling pathways of importance in RA pathogenesis are reviewed in this article. The 'microbiome' (the population of commensals at mucosal surfaces) is also increasingly recognized to play a potential role in the pathogenesis of $R A$.
\end{abstract}

\section{Keywords}

Aetiopathogenesis; angiogenesis; anti-citrullinated peptide antibodies; B cell; co-stimulation; cytokine; dendritic cells; rheumatoid arthritis; T cell; microbiome

\section{Key points}

- Rheumatoid arthritis (RA) is a heterogeneous syndrome with multifactorial aetiopathogenesis

- Genetic mutations of HLA-DRB1 alleles account for a large proportion of genetic risk

- Further investigation of the microbiome may give further insight into the aetiology of RA

- A multitude of proinflammatory cytokines contribute to, and are the focus of many therapies for, RA

\section{Genetic factors}

Genetic factors clearly play a role in the aetiopathogenesis of rheumatoid arthritis (RA). ${ }^{1}$ (Figure 1) There is a slight increase in frequency of RA in first-degree relatives of RA patients, especially if they are seropositive for rheumatoid factor (RF). Furthermore, identical twins share RA in about $12-15 \%$ of instances, compared with $1 \%$ of the general population. The relatively low concordance suggests that RA is a polygenic disease, and that non-genetic factors also contribute to disease pathogenesis.

Over 100 risk loci have been described as predisposing to RA, the largest contribution coming from those encoding particular class II human leucocyte antigens (HLA). Here, the allele HLA-DRB1*04 was found to be present in $70 \%$ of white patients with RA, compared with about $30 \%$ of the healthy control population. Further characterization of the HLA locus identified multiple RA risk alleles within HLADRB1, encoding a conserved amino acid sequence at position 70-74, known as the 'shared epitope'. The shared epitope has been widely demonstrated to be associated with erosive, destructive disease, 
and an increased likelihood of developing anti-citrullinated C-peptide antibody (ACPA; also known as anticyclic citrullinated peptide)-positive RA in association with cigarette smoking has been reported.

The molecular mechanisms accounting for the susceptibility to RA inferred by HLA-DRB1 are unclear, but include modifications in antigen binding, resulting in binding of autoantigens or environmental antigens, initiation of disease by specific binding of superantigens to HLA molecules, or modulation of the $\mathrm{T}$ cell repertoire by selection or tolerance.

\section{The microbiome}

The mucosal surfaces of the oral cavity, upper respiratory tract and gut are colonized by commensal microorganisms; this population is known as the microbiome. Aberrations in the microbiome may facilitate developments in innate and adaptive immunity that predispose to RA. ${ }^{3}$ For example, multiple studies have demonstrated an association between RA and periodontitis, often caused by the bacterium Porphyromonas gingivalis. Studies focusing on the gut microbiome in RA have suggested that an expanded presence of intestinal Prevotella copri, together with a reduction in other bacteria, including Bacteroides species, is more common in patients with new-onset RA compared with healthy controls. The true impact of the microbiome on RA pathogenesis is the subject of continuing investigation.

\section{Autoantibodies}

RF is an antibody directed against the Fc portion of human immunoglobulin (Ig) G. It has long been established that patients with high RF titres are more likely to develop extra-articular disease than those negative for RF. Typically, RF is of $\lg M$ isotype, but $\lg G$ and $\lg A$ also occur. They are found in $75-80 \%$ of RA patients at some time during their disease course.

High-titre IgM RF is relatively specific for the diagnosis of RA in the context of a chronic polyarthritis, and was for decades the sole serological criterion widely used to diagnose RA. It has little predictive value in the general population, although the presence of RF, particularly at high levels, can antedate the clinical development of RA. RF can potentiate inflammation by activating Fc receptors on monocytes, inducing the production of proinflammatory cytokines such as tumour necrosis factor (TNF)- $\alpha$. Immune complexes of RF can also activate complement, potentiating inflammation.

Over the last three decades, there has been increasing recognition of the importance of ACPA in the pathogenesis of RA. Unlike RF, which has little specificity for predicting RA in the general population, the presence of ACPA is more specific and sensitive to RA.

Citrullination involves the protein modification by the conversion of an arginine amino acid into a citrulline amino acid residue, through the action of the enzyme peptidyl arginine deiminase; this produces immunogenic epitopes, to which ACPA binds. A wide range of proteins, including vimentin, fibrin and fibrinogen, can undergo citrullination. ACPAs themselves can form immune complexes, leading to inflammation in the same way as RF.

\section{Cellular pathology}

RA is characterized by chronic inflammation of synovial joints with synovial proliferation and infiltration by blood-derived cells, in particular, memory T cells, macrophages and plasma cells (B cells), all of which show signs of activation. Angiogenesis is a feature from the earliest stages of disease. The synovial tissue becomes markedly hyperplastic and locally invasive at the interface between cartilage and bone, usually with progressive destruction of these tissues. This invasive tissue is termed 'pannus'; it comprises mainly lining cells with the appearance of proliferating mesenchymal cells, with very little sublining lymphocytic infiltration. The accompanying destruction of bone and cartilage is mediated by degradative enzymes, notably matrix metalloproteinases. Although RA has its principal manifestation in joints, there is also evidence of systemic involvement, including up-regulation of acute-phase proteins and a variety of extra-articular features. These occur predominantly in patients who are RF-positive and carry the HLADR4 gene.

T lymphocytes are abundant in active RA, comprising $30-50 \%$ of cells in the inflamed synovium. Activated T cells contribute to the regulation of osteoclast activation and thus joint destruction. Costimulation is an essential component of T cell activation in RA pathogenesis (Figure 2). In this, not only is antigen required to interact with the $T$ cell receptor, but an interaction between co-stimulatory molecules on the T cell and antigen-presenting cell is also crucial. ${ }^{4}$ Presentation of antigen to T cells without costimulation leads to T cell anergy and death. Evidence suggests that co-stimulatory molecules are present in excess within the synovium of patients with RA, implying that T cell activation can take place 
without specific antigen. The importance of co-stimulation in RA is evidenced by the efficacy of abatacept (CTLA4-lg), a soluble fusion protein that binds the co-stimulatory molecule CD28, preventing the second activation signal required to activate $T$ cells.

Multiple theories implicate $B$ cells in RA pathogenesis, predominantly through $B$ cell production of $\mathrm{RF}$ in synovial membrane in RA. RF can be taken up by $\mathrm{B}$ cells and presented to $\mathrm{T}$ cells, triggering $\mathrm{T}$ cell activation. It can also induce activation of synovial macrophages to produce TNF- $\alpha$, and RF complexes can activate $B$ cells themselves, perpetuating B-cell-led inflammatory responses. The significance of $B$ cells in the pathogenesis of RA is evidenced by the success of rituximab as a therapy for RA. This monoclonal antibody, targeted to CD20, a surface antigen highly expressed on B cells, induces B cell depletion, to ameliorate active disease.

The role of cytokines

Cytokines are small, short-lived proteins and important mediators of local intercellular communication. They play a key role in integrating responses to a variety of stimuli in inflammatory processes, and an imbalance in proinflammatory and anti-inflammatory cytokines is thought to contribute to RA (Figure 3); proinflammatory cytokine production is up-regulated, and the up-regulation of anti-inflammatory cytokines such as transforming growth factor (TGF)- $\beta$ is insufficient to achieve a balance. ${ }^{5}$

The key role of TNF- $\alpha$ in RA pathogenesis is evidenced by the efficacy of anti-TNF- $\alpha$ therapy to treat RA. TNF- $\alpha$ regulates the production of other proinflammatory cytokines (such as interleukin (IL)-1) in rheumatoid synovial tissue. It is produced mostly by macrophages, but also by neutrophils, natural killer cells and activated lymphocytes. TNF- $\alpha$ promotes the activation of synovial fibroblasts, which then produce connective tissue growth factor that induces abnormal activation of osteoclasts, culminating in joint destruction. TNF- $\alpha$ also promotes pannus formation through endothelial cell activation and amplification of chemokine production, so promoting the migration of leucocytes into the synovium.

IL-1 is predominantly produced by monocytes and macrophages. It promotes the activation of $\mathrm{T}$ cells, as well as being a potent inducer of matrix metalloproteinases. The IL-1 receptor antagonist anakinra is approved for use in RA, although it has had less success than anti-TNF therapy.

IL-6 is predominantly produced by macrophages and fibroblast-like synoviocytes. IL- 6 in synovial fluid of RA patients is elevated, and correlates with radiological joint destruction. The monoclonal antibody tocilizumab, specific for the IL-6 receptor, is widely recognized as an effective treatment for RA.

Granulocyte-macrophage colony-stimulating factor (GM-CSF) is produced constitutively by synovial macrophages. This cytokine may be sufficient in itself to activate expression of HLA-DR molecules on antigen-presenting cells, promoting activation of $T$ cells. Clinical trials have already confirmed that blocking GM-CSF with monoclonal antibodies (such as mavrilimumab) can decrease disease severity in RA.

The proinflammatory cytokine IL-17 is produced by a specific subset of T cells (T helper type 17 (Th17) cells). Differentiation of T cells to a Th17 phenotype is thought to require a milieu of multiple inflammatory cytokines (including IL-1, IL-6 and IL-23). IL-17 receptors are expressed on multiple cell types, and induce proinflammatory responses through signalling pathways more aligned to the innate immune system (such as Toll-like receptor signalling), inducing the release of acute-phase reactants, and stimulating the generation of proinflammatory cytokines, such as IL-6. IL-17 is further thought to activate osteoclasts, promoting bone destruction. The availability of monoclonal antibodies to IL-17A

(secukinumab, ixekizumab) or to the IL-17 receptor (brodalumab) has confirmed that IL-17 is of relatively greater importance in the pathogenesis of psoriasis, psoriatic arthritis and ankylosing spondylitis than RA.

Many (but not all) of the cytokines implicated in RA exert their effects through signalling via Janus kinases (JAKs). Key cytokines in RA that use JAK signalling include IL-6, IL-10, IL-12 and IL-23. The JAK family of enzymes comprises four isoforms that work in pairs to mediate signalling of type I/II cytokines. When the cytokine binds to its receptor, a conformational change in the intracytoplasmic tail of the receptor allows docking of a pair of JAK enzymes, which become phosphorylated and activated. The activated JAKs then phosphorylate signal transducers of activators of transcription (STATs). JAKs have been validated as therapeutic targets for RA. The oral JAK1,3-selective inhibitor tofacitinib and JAK1,2selective inhibitor baricitinib are able to ameliorate the symptoms and signs of RA, and reduce structural damage. Other JAK inhibitors are in development. 
The potential cause and progression of rheumatoid arthritis

\section{Induction $\longrightarrow$ Chronicity $\longrightarrow$ Effector cells $\longrightarrow$ Pathology $\longrightarrow$ Outcome}

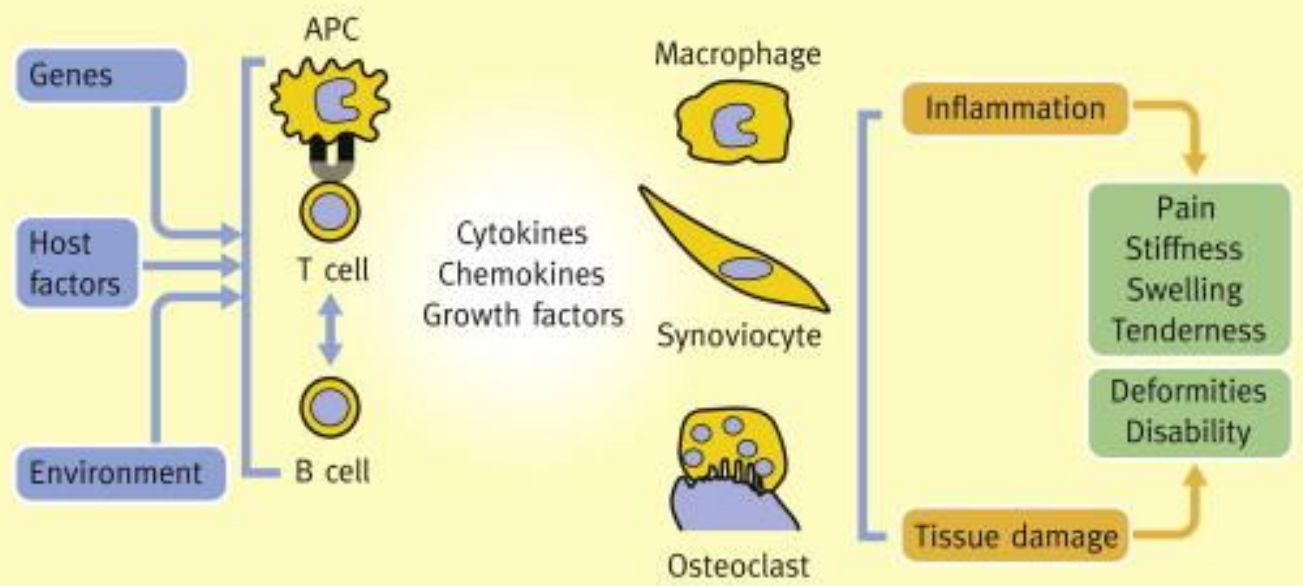

\section{Figure 1}

The potential cause and progression of RA. In genetically susceptible individuals, host and environmental factors can result in activation of pathogenic $T$ cells and a subsequent cascade of inflammatory events that fail to switch off. An example includes the increased occurrence of autoantibodies to certain citrullinated peptides in genetically susceptible individuals who smoke. Cytokines are involved in coordinating the immune response and in the recruitment and activation of effector cells, which results in not only inflammation, but also local tissue damage in the RA joint. APC, antigen-presenting cell. 
STEP 1: TCR recognizes MHC-peptide complex

(signal 1)

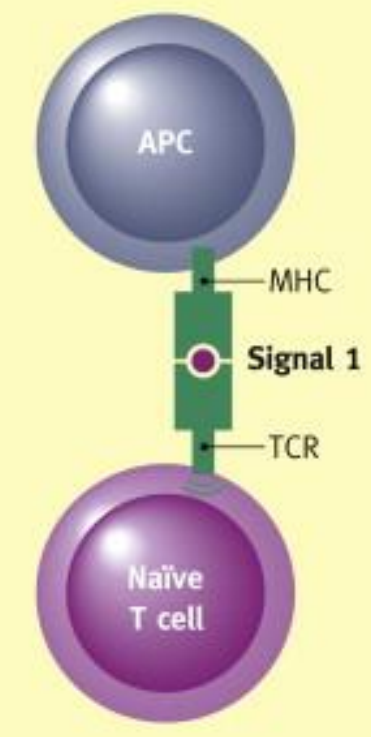

STEP 2: T cell activation follows engagement of an activating co-stimulatory pathway

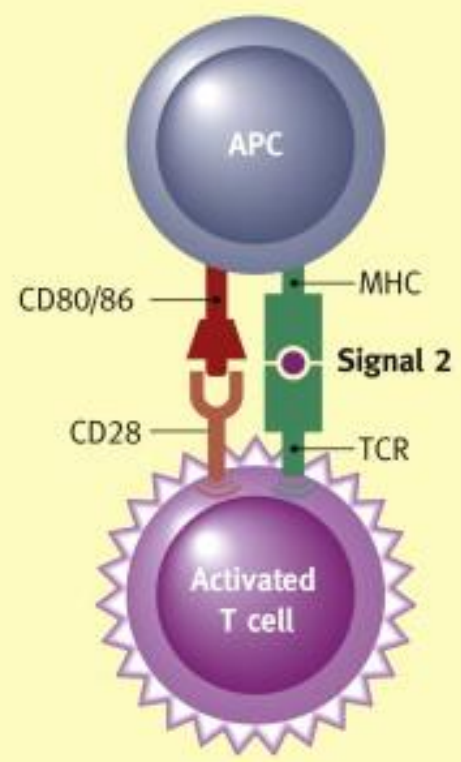

\section{Figure 2}

The T cell receptor (TCR) binds to and recognizes components of both the major histocompatability complex (MHC) and peptide to elicit signal one. There are many different variants of both the $T$ cell receptor and MHC molecules. However, this is insufficient to activate the T cell. Activation follows engagement of a co-stimulatory pathway. CD80/86:CD28 is the best characterized co-stimulatory pathway. CD28 is constitutively expressed on T cells and binds to CD80/86. CD80/86:CD28 facilitates T cell activation, proliferation, survival and cytokine production 


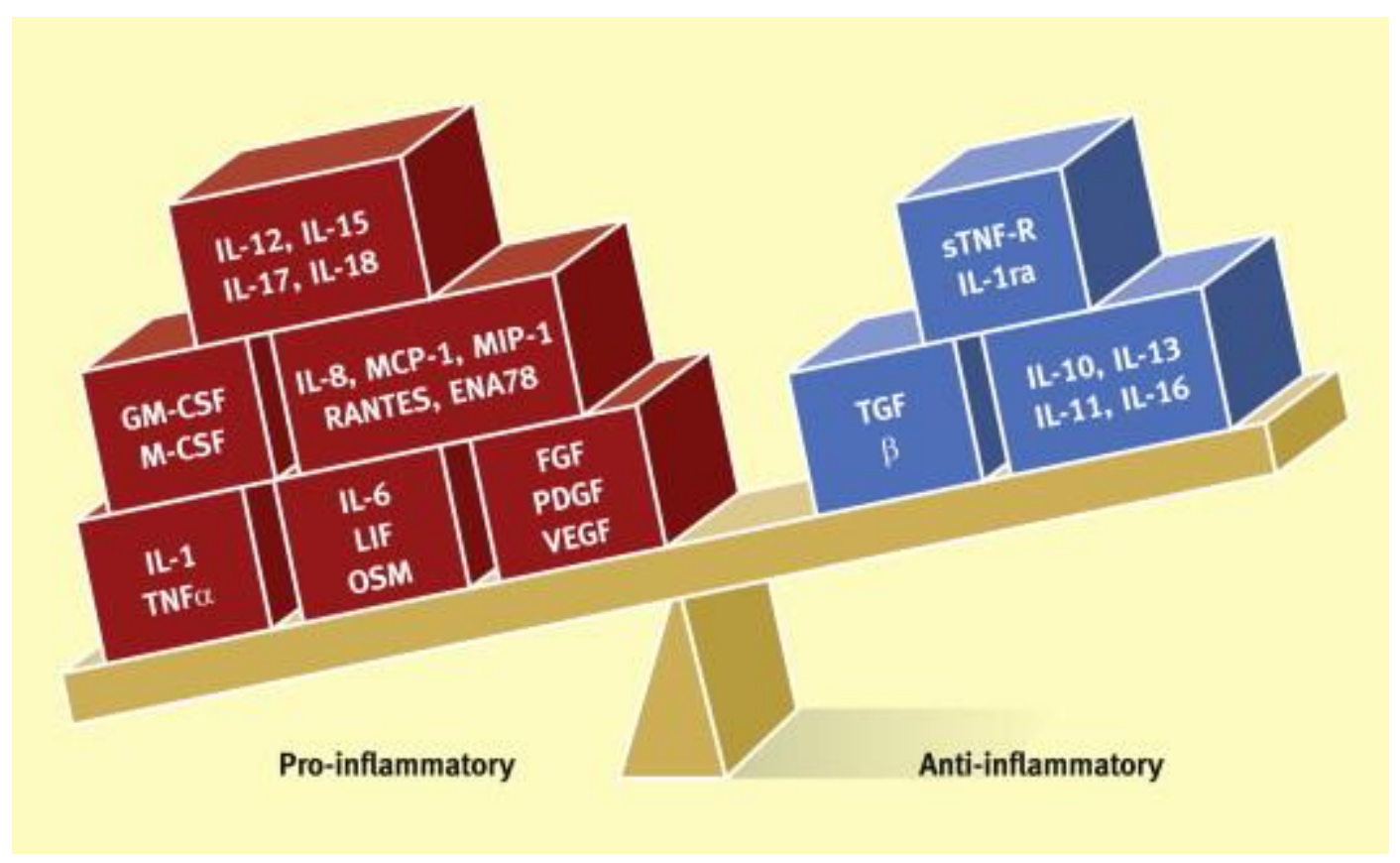

\section{Figure 3}

The concept of a cytokine dysequilibrium. Many cytokines are detectable in rheumatoid synovial tissues, including cytokines with predominantly anti-inflammatory properties, but the net effect is a dominance of proinflammatory activity. ENA, epithelial neutrophil-activating peptide; FGF, fibroblast growth factor; IL1ra, IL-1 receptor antagonist; LIF, leukaemia inhibitory factor; M-CSF, macrophage colony-stimulating factor; MCP, monocyte chemotactic protein; MIP, macrophage inflammatory protein; OSM, oncostatin M; PDGF, platelet-derived growth factor; RANTES, regulated on activation normal T expressed and secreted; sTNFR, soluble TNF receptor; VEGF, vascular endothelial growth factor. 


\section{KEY REFERENCES}

1. Yarwood A, Huizinga TW, Worthington J. The genetics of rheumatoid arthritis: risk and protection in different stages of the evolution of RA. Rheumatology 2016; 55: 199-209.

2. Viatte S, Plant D, Raychaudhuri S. Genetics and epigenetics of rheumatoid arthritis. Nat Rev Rheumatol 2013; 9: 141-53.

3. Jethwa $\mathrm{H}$, Abraham $\mathrm{S}$. The evidence for microbiome manipulation in inflammatory arthritis. Rheumatology 2017; 56: 1452-60.

4. Cope AP, Schulze-Koops $\mathrm{H}$, Aringer M. The central role of T cells in rheumatoid arthritis. Clin Exp Rheumatol 2007; 25:S4-11.

5. Furst DE, Emery P. Rheumatoid arthritis pathophysiology: update on emerging cytokine and cytokineassociated cell targets. Rheumatology 2014; 53: 1560-9. 


\section{TEST YOURSELF}

To test your knowledge based on the article you have just read, please complete the questions below.

The answers can be found at the end of the issue or online here.

\section{Question 1}

A 50-year-old White woman presented with a 3-month history of symmetrical swelling, stiffness and pain in her knuckles. She was a smoker.

After clinical examination and history-taking, which is the most sensitive and specific test for the likely diagnosis?
A Rheumatoid factor
B Antinuclear factor
C Anti-citrullinated peptide antibody
D Plain radiographs of the hands and feet
E C-reactive protein

Correct answer: C. ACPA is the only test mentioned that has both a high overall sensitivity and specificity for the detection of rheumatoid arthritis (61.4\% sensitivity and $89.9 \% 64.5$ specificity for anticitrullinated peptide antibody, compared with $64.4 \%$ sensitivity and $76.5 \%$ for rheumatoid factor).

\section{Question 2}

A 38-year-old man with established, active rheumatoid arthritis attended for his first infusion of tocilizumab.

\section{What is the mode of action of this therapy?}

A Monoclonal antibody binding to IL-17 receptor

B Polyclonal antibody binding to IL-6 receptor

C Inhibition of Janus kinases

D Monoclonal antibody binding to IL-6 receptor

E Inhibition of $T$ cell co-stimulation

Correct answer D. Tocilizumab is a monoclonal antibody binding to the IL-6 receptor, blocking proinflammatory IL-6 signalling. Secukinumab and Ixekizumab are monocloncal antibodies to IL-17 receptor. Tofacitinib and baricitinib are examples of Janus kinase inhibitors in use for the treatment of rheumatoid arthritis. Abatacept is a soluble fusion protein that binds the co-stimulatory molecule CD28, preventing the second activation signal required to activate $T$ cells.

\section{Question 3}

A 62-year-old man presented with a blood result showing a rheumatoid factor mildly positive at $30 \mathrm{IU} / \mathrm{ml}$. He had had this performed at an independent laboratory because of anterior left knee pain on climbing stairs.

What is the most appropriate advice to give?

A This result confirms he has rheumatoid arthritis requiring immediate treatment

$B$ This result is not specific or sensitive for rheumatoid arthritis given his presenting symptoms

C This test confirms he has rheumatoid arthritis currently requiring no specific treatment

$\mathrm{D} \mathrm{He}$ is at risk of rheumatoid arthritis, and requires monitoring for further evidence of inflammatory arthritis

E Further peripheral blood tests for rheumatoid arthritis are required

Correct answer: B. In the absence of chronic polyarthritis, as in this case, rheumatoid factor has little predictive value for rheumatoid arthritis. Given the presenting symptoms, which are more typical of 
osteoarthritis, no further peripheral blood investigations (E), follow-up (C, D) or treatment (A) for rheumatoid arthritis are warranted. 